Proc. Indian Acad. Sci. (Chem. Sci.), Vol. 112, No. 2, April 2000, pp. 109-118

(C) Indian Academy of Sciences

\title{
Synthesis of cadmium sulphide in pure and mixed Langmuir-Blodgett films of $\boldsymbol{n}$-octadecylsuccinic acid
}

\author{
G HEMAKANTHI, BALACHANDRAN UNNI NAIR and \\ ARUNA DHATHATHREYAN* \\ Chemical Laboratory, Central Leather Research Institute, Adyar, \\ Chennai 600 020, India \\ e-mail: adhatha@indiaserver.com
}

MS received 4 December 1999; revised 9 March 2000

\begin{abstract}
Cadmium sulphide (CdS) nanoparticles were grown by the reaction of sodium sulphide $\left(\mathrm{Na}_{2} \mathrm{~S}\right)$ with Langmuir-Blodgett (LB) films of cadmium salts of $n$ octadecylsuccinic acid (ODSU) and with LB films of ODSU in mixtures of octadecylamine and octadecyl alcohol. The results indicate that heterogeneous nucleation and aggregation in the pure ODSU LB films due to processes like Ostwald ripening are destabilized by the presence of the long-chain amine and alcohol in mixed systems. CdS nanoparticles in the LB films were monitored by UV-visible absorption spectra, which allow an estimation of the size of the particles. The morphology, size and nature of the nanocrystallites formed depend on whether the sulphidation was done on the pure film or in the mixed films. It is seen that particles of size around $1.6 \mathrm{~nm}$ were formed in ODSU/octadecylalcohol and ODSU/ octadecylamine mixed LB films while in pure ODSU films the size was about $2.7 \mathrm{~nm}$. These films showed typical needle-shaped structures, as observed by the optical microscopic technique. Mean size and morphology were confirmed by transmission and scanning electron microscopy, while selective area electron diffraction patterns showed six-fold symmetry and indicated that the CdS crystals grow epitaxially with respect to the monolayer. Further, the crystallisation enhanced in the mixed LB films showed a characteristic zinc oxide (Wurtzite) structure compared with the pure ODSU matrix.
\end{abstract}

Keywords. CdS; LB films; octadecylsuccinic acid; epitaxy.

\section{Introduction}

It has been demonstrated that long-chain amphiphiles can be utilized as substrates for nucleation and growth of inorganic and organic crystals and that geometrical and stereochemical matching of the monolayers and crystal lattices can result in epitaxial growth ${ }^{1-3}$. Investigation into epitaxial crystal growth under monolayers has provided insight into such processes as biomineralisation ${ }^{4,5}$ and crystal nucleation growth mechanism ${ }^{6-9}$ and can be expected to produce technologically important devices. The technology of ordered organic LB films gives many opportunities to control the size of $2 d$ semiconductor nanoparticles as has been demonstrated ${ }^{10-16}$. Increasing interest in quantum size II-VI semiconductor systems has been stimulated by their possible

\footnotetext{
*For correspondence
} 
applications in electronics. For instance, single-electron tunneling through $\mathrm{CdS}$ nanoparticles in fatty acid LB film at room temperature has been recently demonstrated ${ }^{17}$. In this work, an investigation into the controlled growth of CdS in LB films of ODSU and mixed films of ODSU with octadecylamine and octadecyl alcohol is described using the reaction with $\mathrm{Na}_{2} \mathrm{~S}$. The nanoparticles so formed were characterized using UV-visible absorption spectra and the morphology characterised using scanning electron microscopy (SEM), selective area diffraction in transmission electron microscopy (TEM) and optical microscopy.

\section{Experimental details}

$n$-Octadecylsuccinic acid (ODSU), octadecylamine (ODAM) and octadecyl alcohol (ODAL) were obtained from Sigma Chemie and were more than 99\% pure. Monolayers of these compounds were formed by spreading millimolar solutions of compounds in chloroform (HPLC grade) on the surface of water (Millipore milli-Q system) containing $5 \times 10^{-4} \mathrm{M} \mathrm{CdCl}_{2}$. The nominal $p \mathrm{H}$ of the subphase was $5 \cdot 5$ (using $\mathrm{NaHCO}_{3}$ ). A NIMA 611 rectangular trough with a Wilhelmy balance and thermostat attached was used for the formation of the monolayer, measurement of $\pi-A$ isotherms and the LB film transfer. The substrates used for SEM and optical micrographs were quartz plates cleaned with chromic acid and then rinsed several times with deionised distilled water while for TEM , carbon coated copper grids were used. A Zeiss-Jena optical microscope was used for the optical microscopic studies. The SEM analyses were carried out using a JEOL scanning microscope JEOM 5500 and $225 \AA$ thick Au films were coated on the LB films. The films were transferred on to carbon-coated copper grids (mesh size 400) using the LB technique, washed with chloroform, and dried with a stream of nitrogen. Selected area diffraction showed ordered domains which were obtained by the small area method using a Philips STEM equipment with a point-to-point resolution of $0 \cdot 1 \mu$. The diffraction patterns were obtained under low-dose conditions in order to reduce sample damage due to radiation. The films were transferred at $\pi=25 \mathrm{mN} / \mathrm{m}$ and the transfer was of the Ytype with a ratio of 0.95 . The formation of CdS in the LB films was achieved by placing the slides in a closed jar containing $\mathrm{Na}_{2} \mathrm{~S}$ in isopropanol for $3 \mathrm{~h}$. Absorption spectra of the LB films were recorded both before and after exposure to $\mathrm{Na}_{2} \mathrm{~S}$ using a Shimadzu UV $160 \mathrm{~A}$ spectrophotometer. The spectra were then subtracted and transformed to $\left((k \times d)^{2} / N\right)$ vs quantum energy $(\mathrm{eV})$ where $k$ is the absorption coefficient near the edge, $d$ is the film thickness estimated from the CPK model and $N$ is the number of layers.

\section{Results and discussion}

The $\pi-A$ isotherms of the pure ODSU on water and $\mathrm{Cd}^{2+}$ containing subphase and with ODSU/ODAM and ODSU/ODAL (1:1 mol ratio) are shown in figures $1 \mathrm{a}$ and $\mathrm{b}$ respectively. Values of the ratio area/molecule of 0.52 and $0.39 \mathrm{~nm}^{2}$ for pure ODSU on water and on $\mathrm{Cd}^{2+}$-containing subphase are in good agreement with estimated crosssectional areas $\left(0.46 \mathrm{~nm}^{2}\right.$ and $\left.0.40 \mathrm{~nm}^{2}\right)$ using CPK models. The large plateau has been attributed to the inequivalent nature of the two carboxylic acid groups ${ }^{18}$ in long-chain carboxylic acid monolayers. In case of the mixed layers there is a slight expansion in area $\left(0.50 \mathrm{~nm}^{2}\right)$ for both alcohol- and amine-containing monolayers on $\mathrm{CdCl}_{2}$ subphase. It was observed that when the ODSU concentration was maintained higher than that of ODAM or ODAL, the isotherms of the mixed monolayers became indistinguishable from the pure 

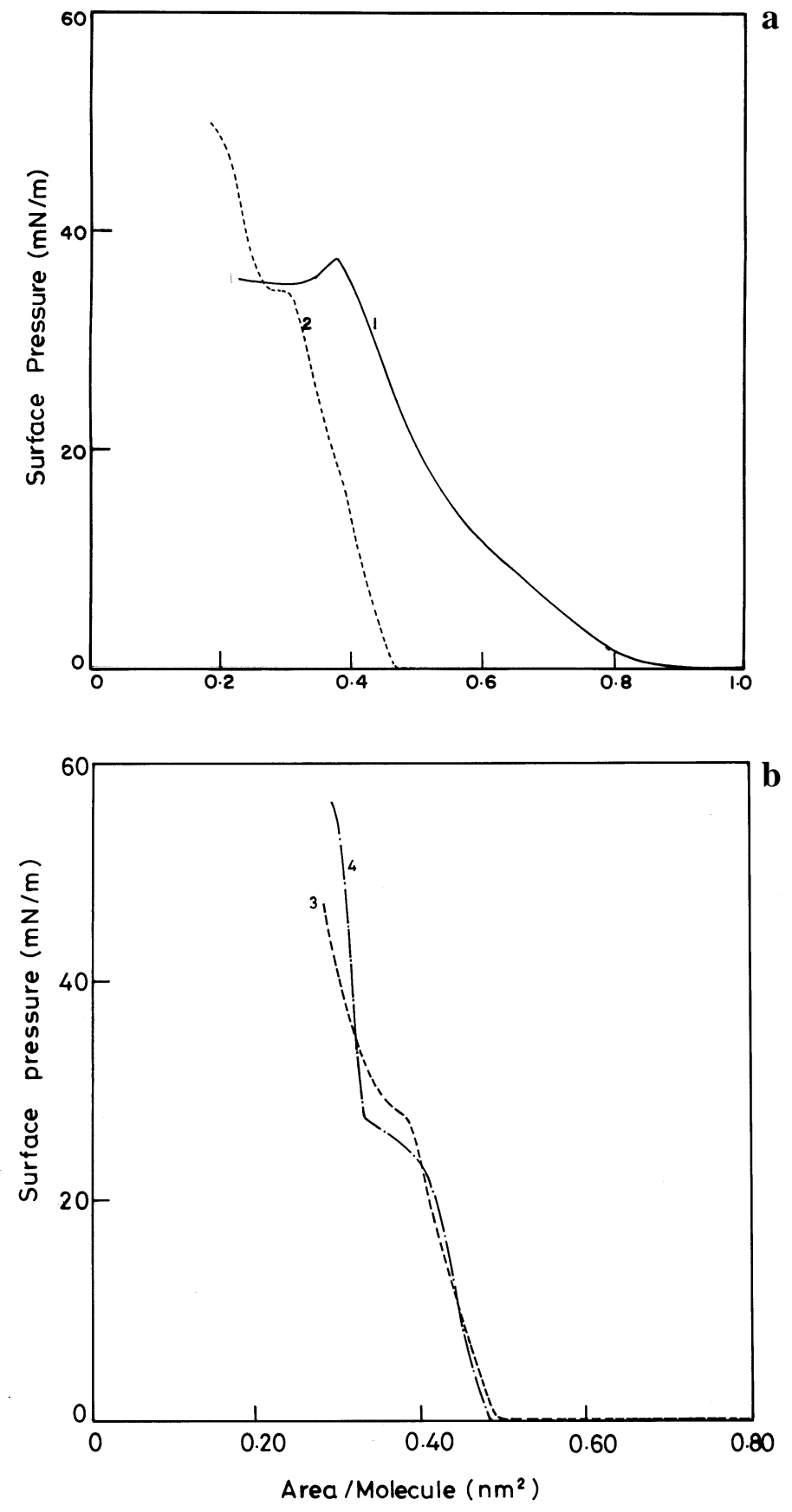

Figure 1. $\pi-A$ isotherm of (a) ODSU on (1) pure water and (2) $\mathrm{CdCl}_{2}$ containing subphase $(p \mathrm{H}=5 \cdot 5)$, and (b) ODSU/ODAM and ODSU/ODAL (1:1 mol ratio) on $\mathrm{CdCl}_{2}$ containing subphase $(p \mathrm{H}=5 \cdot 5)$. 
ODSU film (for instance 2:1, 5:1, etc.). These results imply that ODAM or ODAL when present in the mixed layer in amounts smaller than those of the pure component adopt the compression-dependent structure of the ODSU matrix. This may be due to the possible $\mathrm{H}-$ bond formed between the $-\mathrm{OH}$ and the $-\mathrm{NH}_{2}$ groups and the carboxylic groups in these mixed films. This is also confirmed by the lowering of the surface pressure at which the plateau appears, as well as the shortening of the plateau itself.

\subsection{Optical absorption spectra}

The differences in the UV-visible absorption spectra of the LB films of ODSU and ODSU/ODAM and ODSU/ODAL of the $\mathrm{Cd}^{2+}$ salt after sulfidation are shown in figure 2 . It should be noted that the absorption edge of CdS particles formed in fatty acid LB films is usually observed in the range $450-500 \mathrm{~nm}$, while that of the edge of the bulk CdS material is near $515 \mathrm{~nm}$. The fact that the absorption onset, that is the dimension of $\mathrm{CdS}$ formed is identical irrespective of the number of layers (experiments were carried out with $n=2$ to 20 layers) demonstrates that controlled formation of CdS takes place within each hydrophilic interlayer and that the CdS formed remains within the interlayers. The absorption spectra exhibited Beer-Lambert behaviour (as seen from the absorption as a function of number of layers, not shown here for the sake of brevity). Peak positions are not very clear, especially in the pure ODSU film, probably due to wide particle size distribution.

CdS particles generated in the matrix of the amphiphiles can be described as 3-d quantum wells. The motion of electrons, holes or excitons in such a well being limited, leads to quantization of kinetic energy. Hence, in principle, the nanosized CdS obtained from such LB films should give rise to a series of separate lines. However there being a dispersion of size of the particles and electron-phonon interactions, broad bands are seen in the optical absorption spectra. Light absorption corresponds to electron transitions

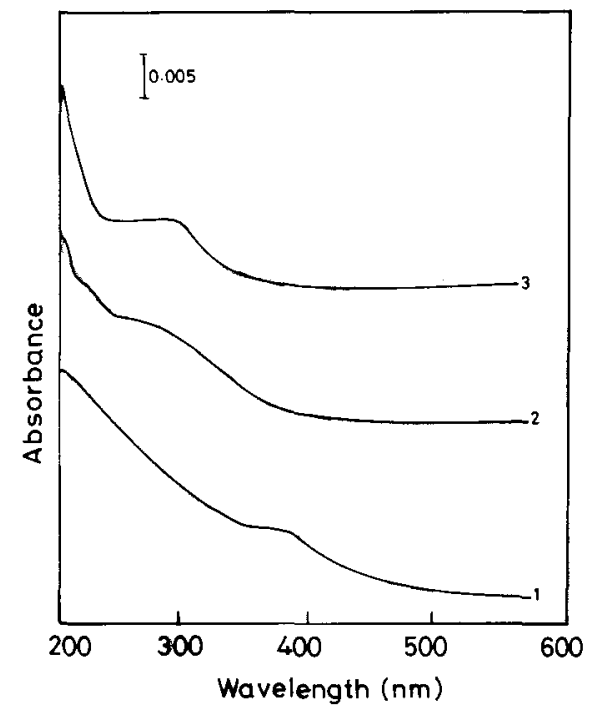

Figure 2. UV-visible absorption spectra (difference) of (1) pure ODSU, (2) ODSU/ODAM, ODSU/ODAL, (3) mixed LB films (Cd salt of the film) obtained by subtraction of corresponding spectra after and before exposure to $\mathrm{Na}_{2} \mathrm{~S}$. 

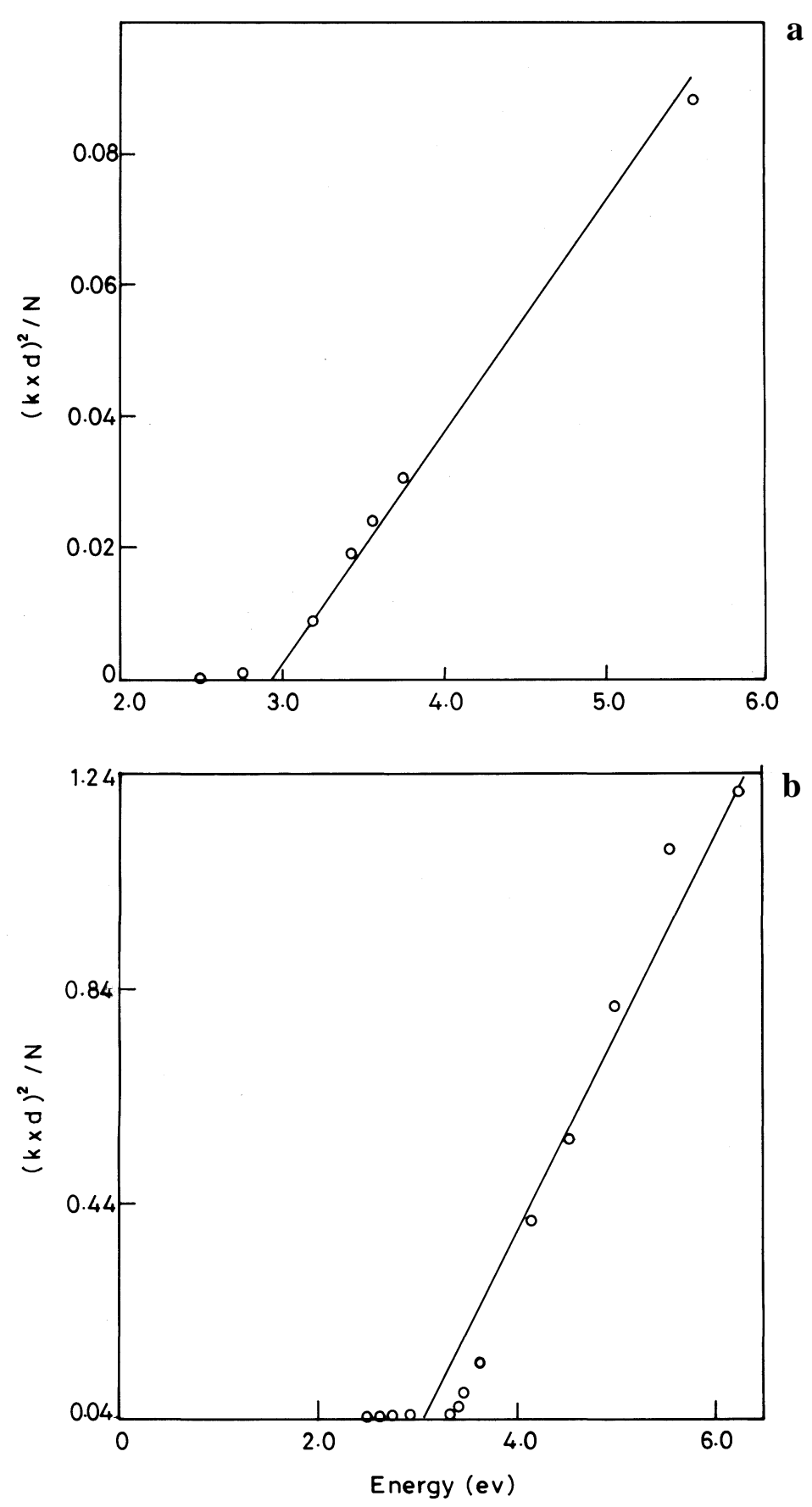

Figure 3. (a) and (b). (Continued, for caption see next page.) 


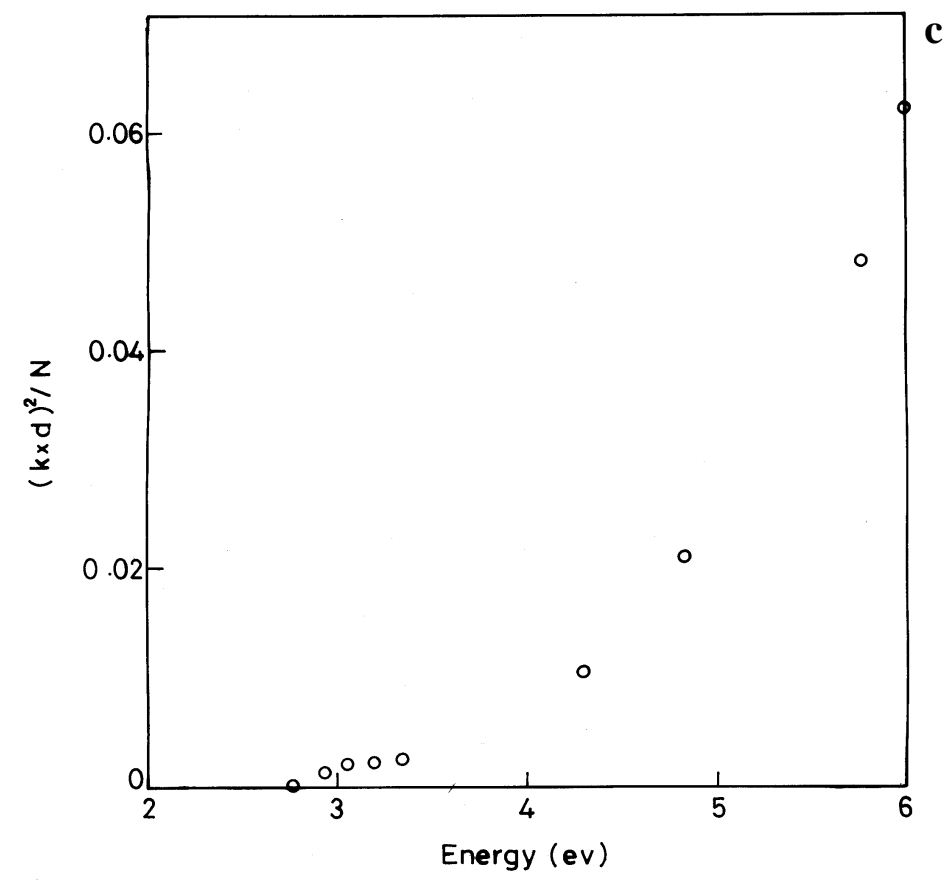

Figure 3. Part of the spectra showing edge of the absorption: Cd salt of (a) ODSU, (b) ODSU/ODAM, (c) ODSU/ODAL (after formation of CdS).

between levels of size-quantization for electrons $E_{l, n}^{e}$ and holes $E_{l, n}^{h}$ with $l, n=$ constant. The position of the absorption band then can be written ${ }^{19}$ as

$$
h v_{01}=E g+\left(h^{2} / 8 \pi^{2} \mu a^{2}\right) J_{l, n}{ }^{2},
$$

where $\mu=m_{e} m_{h} /\left(m_{e}+m_{h}\right)$ and $a$ is the size of the particle. Here the $J$ values are the roots of the Bessel functions with $l$ and $n$ being the angular and spin quantum numbers. The absorption coefficient near the edge can be derived as

$$
k=A(h v-E g)^{1 / 2},
$$

where $A$ is a constant ${ }^{20}$.

The plot of $\left((k \times d)^{2} / N\right)$ as a function of the energy is shown in figures 3a-c. The absorption edge for $\mathrm{CdS}$ in pure ODSU is equal to $2.9 \mathrm{eV}$, while in the mixed ODSU/ODAM it is $3.1 \mathrm{eV}$. The value of $2.9 \mathrm{eV}$ is very similar to those obtained for group II-VI semiconductor nanoparticles in fatty acid LB films ${ }^{21}$. The particle sizes range between 1.6 and $2.0 \mathrm{~nm}$ while for pure ODSU the size is around $2.7 \mathrm{~nm}$.

\subsection{Optical and electron micrographs}

The optical micrographs in figure 4a show needle-shaped CdS crystallites with occasional clusters in pure ODSU LB film. In the case of mixed films (figure $4 \mathrm{~b}$ ) clear crystallites 


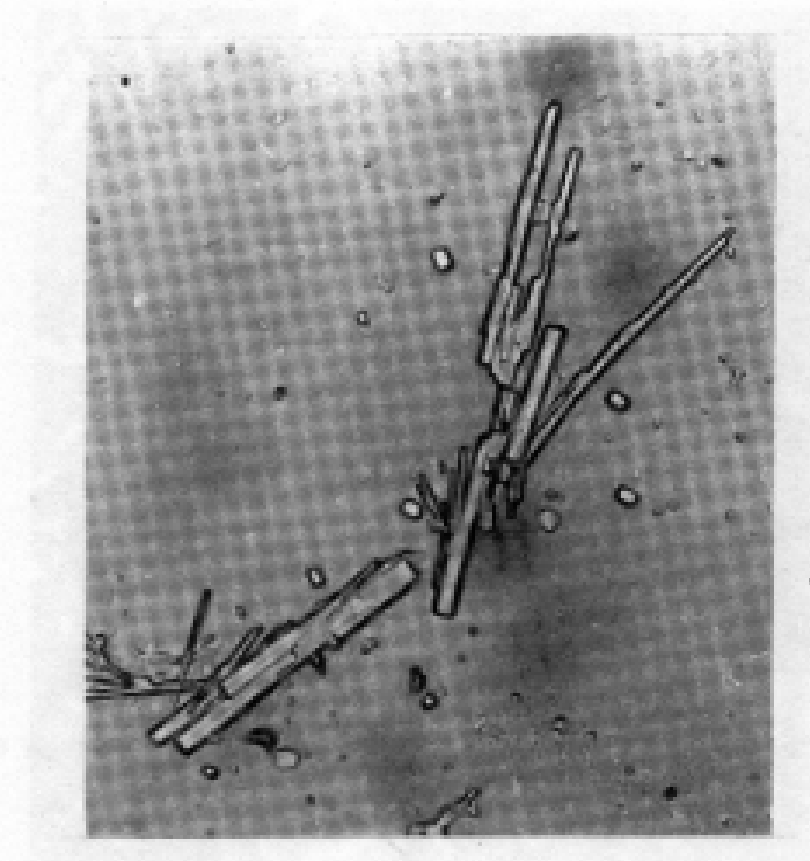

(a)

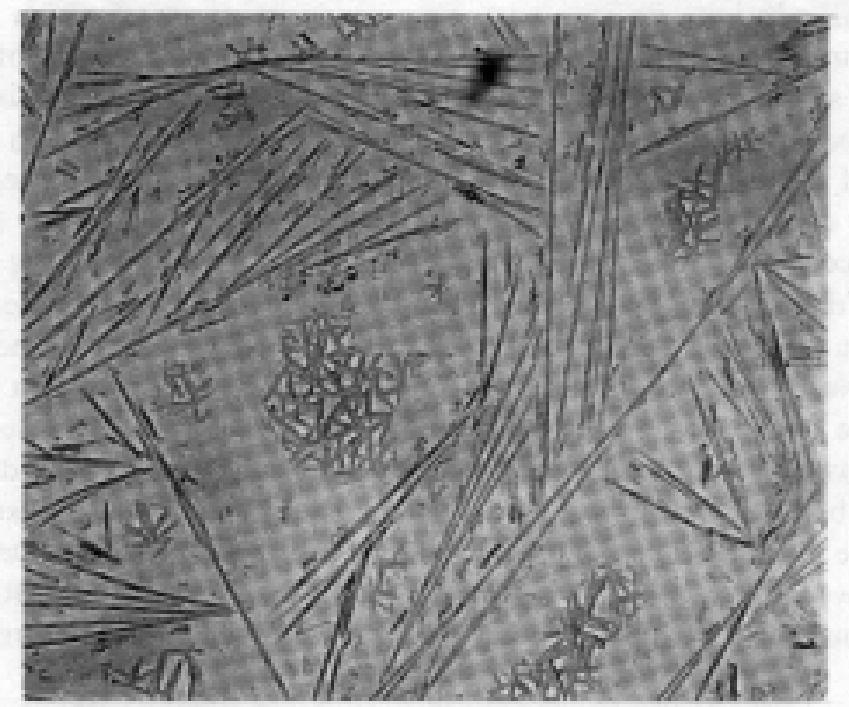

(b)

Figure 4. Optical micrographs of CdS crystallites in (a) the LB films of ODSU, and (b) mixed films containing ODSU/ODAM (scale $1 \mathrm{~cm}=1 \mu$ ).

are seen to grow. The scanning electron micrograph of gold-coated LB films of mixed ODSU/ODAM film (figure 5) shows highly clustered crystals in mixed film. TEM micrographs obtained from the bright field mode are shown in figure 6. In the mixed films the isolated crystals possess rodlike morphologies of lengths 5-30 nm and width $2-5 \mathrm{~nm}$, 


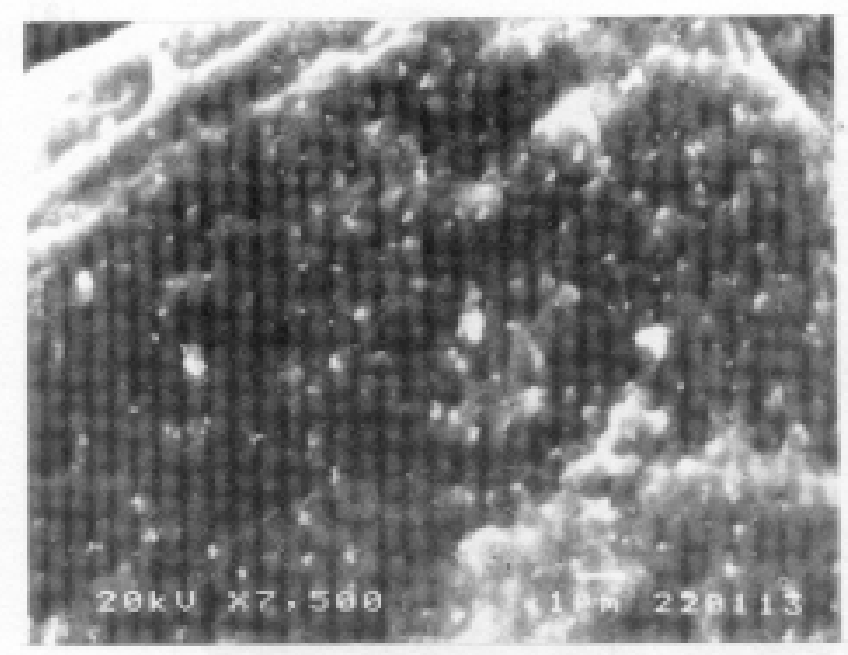

Figure 5. Scanning electron micrographs showing typical morphology of the CdS crystallites in mixed LB film.

while in the pure film rod-like crystals with more dendritic outgrowths from the major axis are seen. Electron diffraction of the selected area using a $2-\mu \mathrm{m}$ aperture of the same area show six-fold symmetry. That the patterns display distinct reflections as opposed to being the powder pattern type confirm that the crystal growth is epitaxial with respect to the monolayer and demonstrate that this lattice correspondence is maintained even in regions of denser crystal coverage. Hexagonal $\mathrm{CdS}$ possesses zinc oxide (Wurtzite) structure of lattice constant $a=4.136 \AA$ and $c=6.713 \AA$. The results obtained demonstrate that $\mathrm{CdS}$ nanoparticles in the size range 2-5 nm are formed in the matrix of $n$-octadecylsuccinic acid and mixtures of ODSU with long-chain amines and alcohols after exposure to $\mathrm{Na}_{2} \mathrm{~S}$. The particle size is notably less in the mixed films. In the mixed LB films, there are two processes in the growth of crystallites at the interface that has been earlier observed: Ostwald ripening and aggregation ${ }^{22}$. During Ostwald ripening, particles grow via bond formation of source ions with their surface atoms, resulting in a continuous increase of the particle size. This would be true for CdS $>2 \mathrm{~nm}$. On the other hand, reaction between any two nanoparticles can lead to stable aggregates. Reactivities of the surface atoms of $\mathrm{CdS}$ with $\mathrm{Cd}^{2+}$ or $\mathrm{S}^{2-}$ play an important role in determining the final size.

In the present study, the H-bonding between neighbouring ODSU and between ODSU and ODAM/ODAL contribute to the organisation and structural integrity of the mixed layer, thereby further reducing the mobility of the $\mathrm{Cd}^{2+}$ ions. The mobility of $\mathrm{Cd}^{2+}$ ions and of the formed CdS in the less-ordered film of ODSU is higher than in the wellordered mixed films, which makes the growth of CdS leading to larger clusters easier. The smaller size and the narrow size distribution of CdS obtained in the mixed films indicate little Ostwald ripening and aggregation. Thus, morphological differences imply that repeated homogenous nucleation of CdS in ODSU/ODAL or ODSU/ODAM occurs but that similar homogenous nucleation in the pure film is difficult. In the latter case, after the initial nucleation, further growth involves heterogeneous nucleation and growth of 


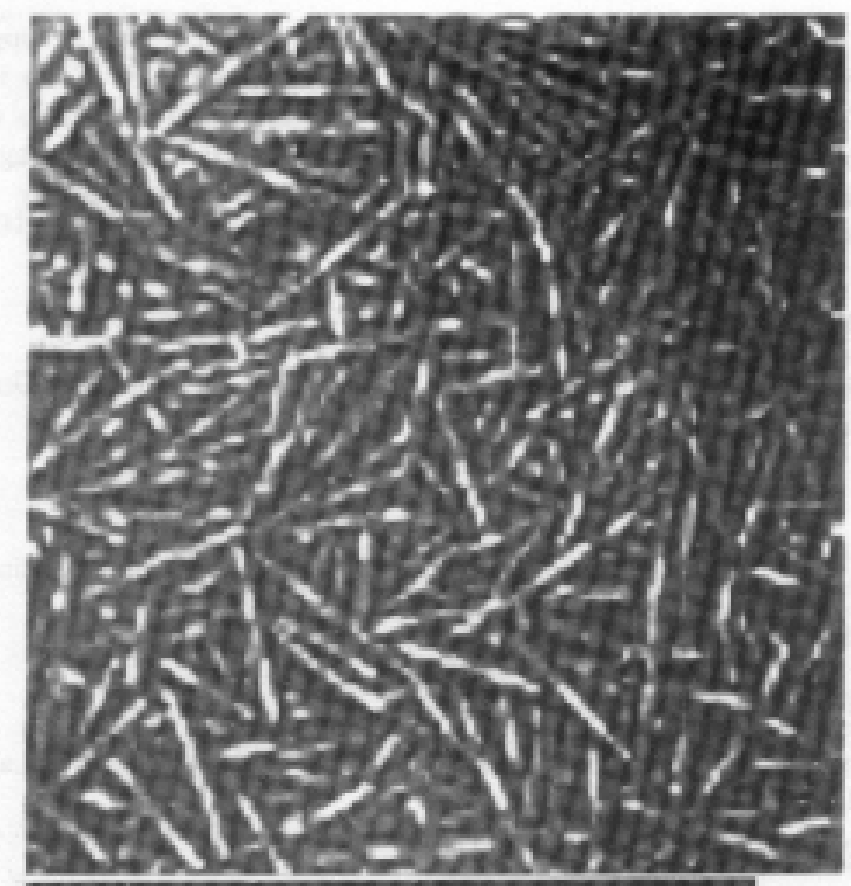

(a)

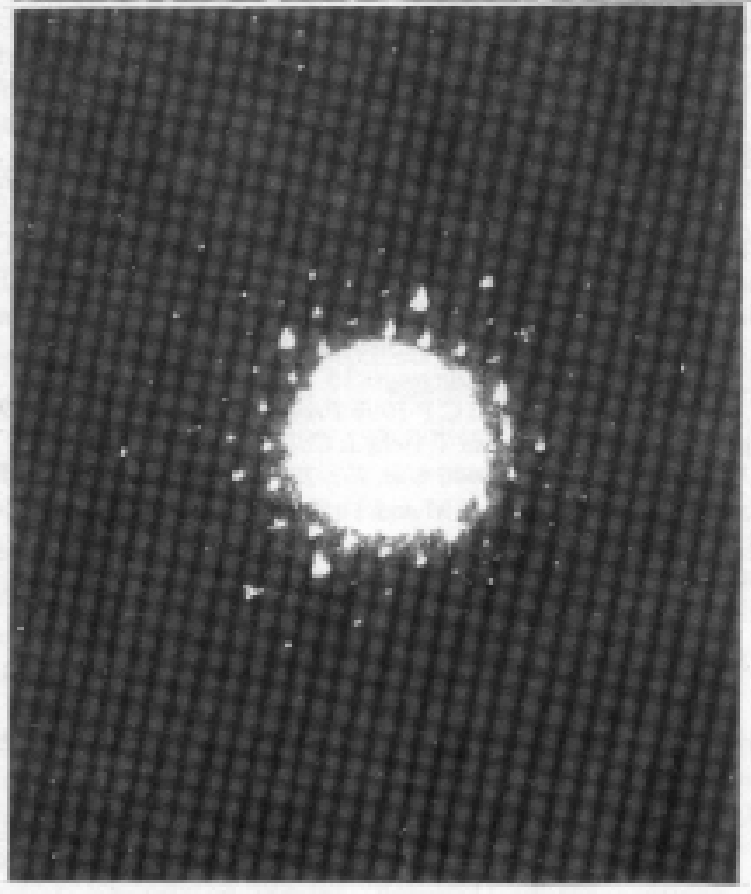

(b)

Figure 6. (a) Dark field image of the CdS crystallites (scale $1 \mathrm{~cm}=25 \mathrm{~nm}$ ), and (b) selective area electron micrographs [110] zone axis patterns of the zinc oxide structure of the crystallites. 
additional CdS particles on the surface of existing small particles. This is supported by the smaller CdS particle density. This difference would be expected if the interfacial energy of the CdS/ODSU is significantly larger than for the CdS/mixed films case. The optical and electron micrographs show that the mean size, particle size and aggregation play considerable roles in synthesizing nanocrystallites showing epitaxy with the underlying monolayers.

\section{Acknowledgment}

The authors would like to thank the Department of Science and Technology, Government of India for a project grant for this work.

\section{References}

1. Fendler J H 1994 Membrane mimetic approach to advanced materials (Berlin: Springer Verlag)

2. Yang J, Meldrum F C and Fendler J H 1995 J. Phys. Chem. 995500

3. Yang J and Fendler J H 1995 J. Phys. Chem. 995505

4. Heywood B R and Mann S 1994 Adv. Mater. 69

5. Mann S 1993 J. Chem. Soc., Dalton Trans. 1

6. Majewski J, Margulis L, Jacqemain D, Leveiller F, Bohm C, Arad T, Talmon Y, Lahav M and Leiserowitz L 1993 Science 261899

7. Landau E M, Wolf S G, Levanon M, Leiserowitz L, Lahav M and Sagiv J 1989 J. Am. Chem. Soc. 1111436

8. Majewski J, Popovitz-Biro R, Kjaer K, Als-Nielsen J, Lahav M and Leiserowitz L 1994 J. Phys. Chem. 984987

9. Landau E M, Popovitz-Biro R, Levanon M, Leiserowitz L and Sagiv J 1986 J. Mol. Cryst. Liq. Cryst. 134323

10. Nabok A V, Richardson T, Davis F and Stirling C J M 1997 Langmuir 133198

11. Guo S, Popovitz-Biro R, Weissbuch I, Cohen H, Hodes G and Lahav M 1998 Adv. Mater. 10 121

12. Dekany L, Nagy L, Turi L, Kiraly Z, Kotov N A and Fendler J H 1996 Langmuir 123709

13. Gao M, Yang Y, Yang B, Bcan F and Shen J 1994 J. Chem. Soc., Chem. Commun. 2777

14. Kang Y S, Risbud S, Rabolt J and Stroeve P 1996 Langmuir 124345

15. Ogava S, Fan F R F and Bard A J 1995 J. Phys. Chem. 9911182

16. Hemakanthi G and Dhathathreyan A 1999 Langmuir 153317

17. Facci P, Erokhim V, Carrara S and Nicolini C P 1996 Proc. Natl. Acad. Sci. USA 9310556

18. Collins S J, Dhathathreyan A and Ramasami T 1998 J. Coll. Interf. Sci. 179357

19. Kulish N R, Kunetz U P and Lisitsa M P 1990 Ukr. Fiz. Zh. (Russian edn) 351817

20. Narayanan K L, Vijayakumar K P, Nair K G M and Thampi N S 1997 Physica B 2408

21. Kang Y S, Risbud S, Rabolt J and Stroeve P 1996 Langmuir 124345

22. Dhathathreyan A and Ramasami T 1997 Philos. Mag. Lett. 7699 